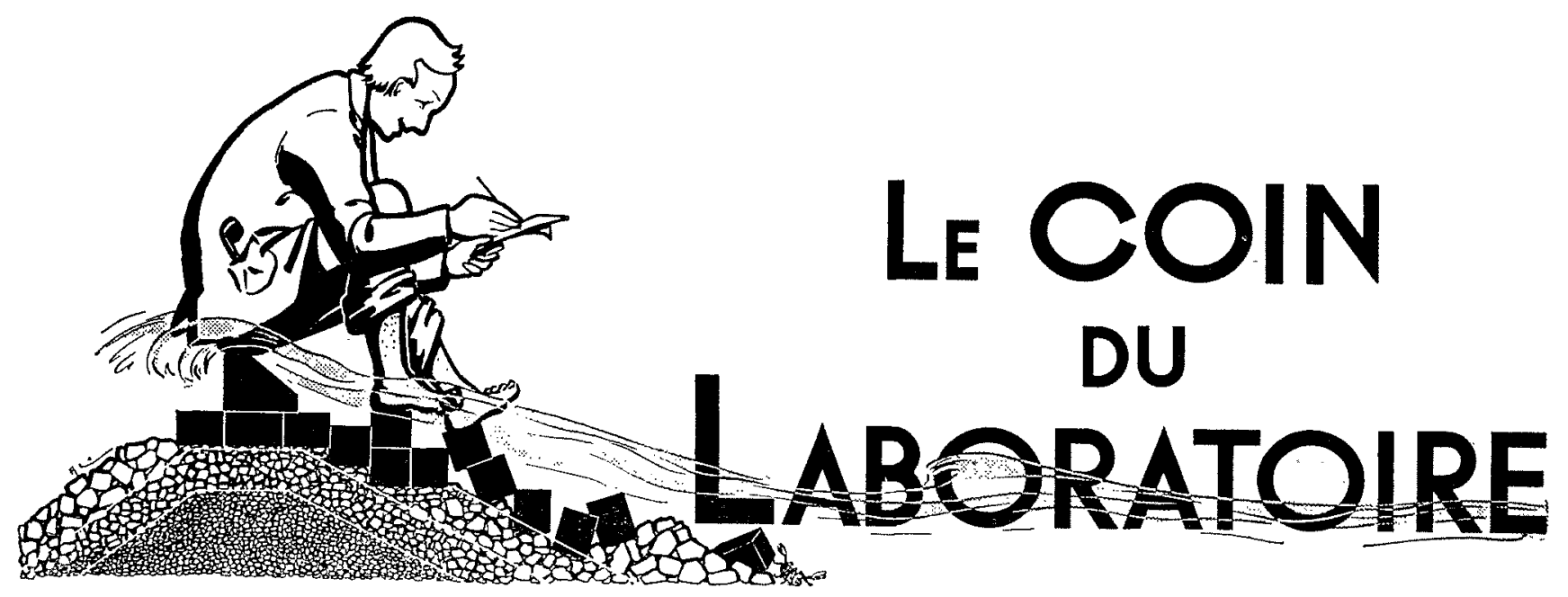

\title{
La visualisation des écoulements entre deux plaques parallèles rapprochées (application aux milieux poreux)*
}

\author{
Visual observation of flow \\ between two parallel plates placed close together, \\ and its application to porous media
}

PAR

B. LE FUR,

DOCTEUn Ès SCrENCES, INGÉNIEUR DE RECHERCHES

A L'INSTITUT'T FRANÇATS DU PÉTRORE
La visualisation des écoulements dans les milieux porenx nécessite habituellement des modèles transparents remplis de billes de verre. On peut éviter la construction de ces modèles coûteux et difficiles d transformer, en utilisant l'analogie avec l'écoulement, à faible nombre de Reynolds, entre deux plaques parallèles.

Principe d'un appareil qui permet de visualiser ce qui se passe dans une couche perméable horizontale, lorsqu'on $y$ injecte par un puits un fluide moins visqueux que le fluide en place, ce fluide pouvant être miscible ou non.

\section{I. - INTRODUCTION}

On sait que la vitesse d'un fluide incompressible dans un milieu poreux dépend d'un potentiel harmonique. Si l'écoulement est bidimen-

\author{
J. AGUIRRE-PUENTE, \\ MNGÉNIEUR-DOCTEUR \\ ASSISTANT A LA FACULTË DES SCIENCES
}

sionnel, on peut utiliser une analogie basée sur l'écoulement d'un fluide visqueux entre deux

- Ce travail a été effectué au Laboratoire d'Aćrothermique dn C.N.R.S. dans le cadre des recherches de l'Association de Recherche sur les Techniques de Forage et de Production. 
plaques parallèles, imaginée par Hele-Shaw en 1898. Cette analogie permet, en outre, d'obtenir par visualisation la forme du front qui séparerait deux fluides non miscibles dans un massif perméable.

\section{II. - PRINCIPE}

\section{DE L'ANALOGIE DE HELE-SHAW}

Pour démontrer l'analogie, nous allons examiner comment les équations de Navier peuvent se simplifier lorsque le nombre de Reynolds basé sur l'écartement $e$ et la vitesse locale reste faible. Les termes d'inertie sont alors négligeables et l'on a, si l'écoulement est stationnaire :

$$
\mu \Delta \overrightarrow{\mathrm{U}}-\overrightarrow{\operatorname{grad}} \hat{p}=0
$$

$\overrightarrow{\mathrm{U}}$ est le vecteur-vitesse, $\mu$ la viscosité et

$$
\widehat{p}=p+\rho g z
$$

la pression motrice, si l'axe vertical des $z$ est dirigé vers le haut.

Si les plaques sont horizontales, nous pouvons prendre comme plan des $x$ et des $y$ le plan médian de l'espace compris entre les deux plaques. Les conditions aux limites auxquelles doit obéir l'équation (1) sont $\overrightarrow{\mathrm{U}}=\widehat{0}$ pour $z= \pm e / 2$.

Si la distance entre les deux plaques est faible devant les dimensions des frontières de l'écoulement, on peut supposer, d'une part, que la composante $w$ de la vitesse le long de l'axe des $z$ est négligeable devant les autres composantes $u$ et $v$, d'autre part, que les dérivées par rapport à $x$ ou à $y$ sont négligeables devant les dérivées par rapport à $z$. Les équations de Navier sous la forme de Stokes (1), se réduisent au système :

$$
\begin{aligned}
\mu \frac{\partial^{2} u}{\partial z^{2}}-\frac{\partial \hat{p}}{\partial x} & =0 \\
\mu \frac{\partial^{2} v}{\partial z^{2}}-\frac{\partial \hat{p}}{\partial y} & =0 \\
\frac{\partial \hat{p}}{\partial z} & =0
\end{aligned}
$$

On voit donc que la pression motrice ne dé- pend que de $x$ et de $y$ et que les composantes de la vitesse sont de la forme :

$$
\left.\begin{array}{l}
u(x, y, z)=u_{\mathrm{M}}(x, y)\left(1-\frac{4 z^{2}}{e^{2}}\right), \\
v(x, y, z)=v_{\mathrm{M}}(x, y)\left(1-\frac{4 z^{2}}{e^{2}}\right),
\end{array}\right\}
$$

avec :

$$
\left.\begin{array}{l}
u_{\mathrm{M}}=-\frac{e^{2}}{8 \mu} \cdot \frac{\partial \hat{p}}{\partial x}, \\
v_{\mathrm{M}}=-\frac{e^{2}}{8 \mu} \cdot \frac{\partial \hat{p}}{\partial y} \cdot
\end{array}\right\}
$$

Lorsqu'on se déplace perpendiculairement aux plaques, l'angle du vecteur-vitesse est invariable et son extrémité décrit une parabole. La vitesse maximale $\overrightarrow{\mathrm{U}}_{\mathrm{M}}(x, y)$, de composantes $u_{\mathrm{M}}$ et $v_{\mathrm{MI}}$, est atteinte sur le plan médian $z=0$.

On peut prendre la moyenne de la vitesse perpendiculairement aux plaques, on aura ainsi :

$$
\overrightarrow{\mathrm{U}}_{m}(x, y)=\frac{1}{e} \int_{-e / 2}^{e / 2} \overrightarrow{\mathrm{U}}(x, y, z) d z=\frac{2}{3} \overrightarrow{\mathrm{U}}_{\mathrm{M}} .
$$

Cette vitesse moyenne est reliée au gradient de pression motrice par l'expression :

$$
\overrightarrow{\mathrm{U}}_{m}=-\frac{e^{2}}{12 \mu} \overrightarrow{\operatorname{grad}} \widehat{p}
$$
tiel :

La vitesse moyenne dépend donc d'un poten-

$$
\Phi=-\frac{e^{2}}{12 \mu} \widehat{p}
$$

L'expression (6) est analogue à la loi de Darcy, la perméabilité étant égale à $e^{2} / 12$.

\section{III. - ECOULEMENT DIPHASIQUE DANS UN MILIEU POREUX}

Lorsqu'un fluide se trouvant dans un milieu poreux est déplacé par un autre fluide non miscible, il en est séparé par une zone où les proportions volumiques des fluides, appelées saturations, varient.

Ceci se produit, par exemple, au cours de la production des gisements de pétrole au moyen d'injection d'eau ou de gaz par certains des puits. 
On admet souvent, pour simplifier les calculs, que le fluide primitivement en place et le fluide injecté sont séparés par un front discontinu à travers lequel on passe brusquement d'un fluide à l'autre.

L'écoulement produit par l'injection d'eau dans une couche horizontale de roche saturée de pétrole peut être simulé à l'aide de l'analogie de Hele-Shaw, en supposant que la simplification précédente a été adoptée. En effet, dans un modèle de Hele-Shaw, le fluide injecté sera, à cause de la capillarité, séparé du fluide primitivement en place par une surface incurvée qui s'appuiera sur les deux plaques.

Nous nous sommes intéressés tout d'abord au cas le plus simple, celui de l'écoulement à des distances d'un puits d'injection faibles devant celles qui le séparent des autres puits. Tout se passe alors comme si le gisement était infini.

Dans l'analogie de Hele-Shaw, le puits sera simulé par un trou percé dans l'une des placues. La composante verticale $w$ de la vitesse ne sera donc pas négligeable au voisinage immédiat du trou, mais plus loin, l'écoulement obéira aux équations (2) de Hele-Shaw.

Lorsqu'on a un seul fluide en présence, la vitesse moyenne est inversement proportionnelle à la distance du trou et la pression motrice est uniforme sur des cylindres circulaires ayant le même axe que le trou. Si l'on injecte, à débit constant, un autre liquide, on devrait avoir théoriquement un front circulaire dont le rayon serait proportionnel à la racine carrée du temps. Les surfaces isobares seraient encore des cylindres circulaires coaxiaux.

Dans le modèle que nous avons construit, nous nous sommes imposé d'avoir une pression motrice uniforme à la limite circulaire de l'espace entre les plaques, afin d'avoir le mème écoulement que dans un gisement indéfini, si le front restait circulaire.

Des expériences d'écoulements unidimensionnels [1] et [2] ont montré que lorsqu'on déplace un fluide dans un milieu poreux ou entre deux plaques parallèles, par un autre fluide moins visqueux, la forme théorique du front, qui est alors plane, était instable. Le front se déformait, certains endroits avancant plus vite que les autres en formant ce qu'on appelle des digitations.

Nous avons voulu voir comment ce phénomène se présentait dans un écoulement divergent, mais il faut souligner que les digitations qui se produiront dans le modèle n'auront pas tout à fait la même forme qu'elles auraient eu entre deux plaques indéfinies, puisque la condition de pression motrice uniforme à la limite de l'espace entre les plaques ne serait évidemment plus vérifiée. L’analogie entre ce qui se passe dans le modèle et ce qui se passe dans le gisement sera la meilleure lorsque le front ne sera ni trop près du trou d'injection, ni trop près de la limite circulaire de l'écoulement.

\section{IV. - DESCRIPTION DU MODELE}

Le modèle où se produisent les écoulements entre deux plaques parallèles a une forme circulaire (photo $n^{\circ} 1$ ). C'est un réservoir constitué de deux disques en verre de $41 \mathrm{~cm}$ de diamètre et de $10 \mathrm{~mm}$ d'épaisseur, et d'une couronne en Iaiton de $36 \mathrm{~cm}$ de diamètre intérieur, de $40 \mathrm{~cm}$ de diamètre extérieur et de $2,5 \mathrm{~cm}$ de hauteur. Deux disques de plexiglas s'appliquent respectivement sur les disques de verre supérieur et inférieur et sont serrés l'un contre l'autre à l'aide de boulons. L'élanchéité du réservoir est assurée par l'écrasement de deux joints toriques en caoutchouc logés dans deux gorges creusées dans l'anneau en laiton.

Avant de poser le disque de verre supérieur sur l'anneau en laiton, on place un disque de verre intermédiaire de $32 \mathrm{~cm}$ de diamètre et de $10 \mathrm{~mm}$ d'épaisseur, au-dessus du disque de verre inférieur et à une distance de $1,3 \mathrm{~mm}$. Cet écartement est obtenu à l'aide de trois cales circulaires en laiton que l'on peut voir sur les photos. Sur le disque intermédiaire, sont posés trois

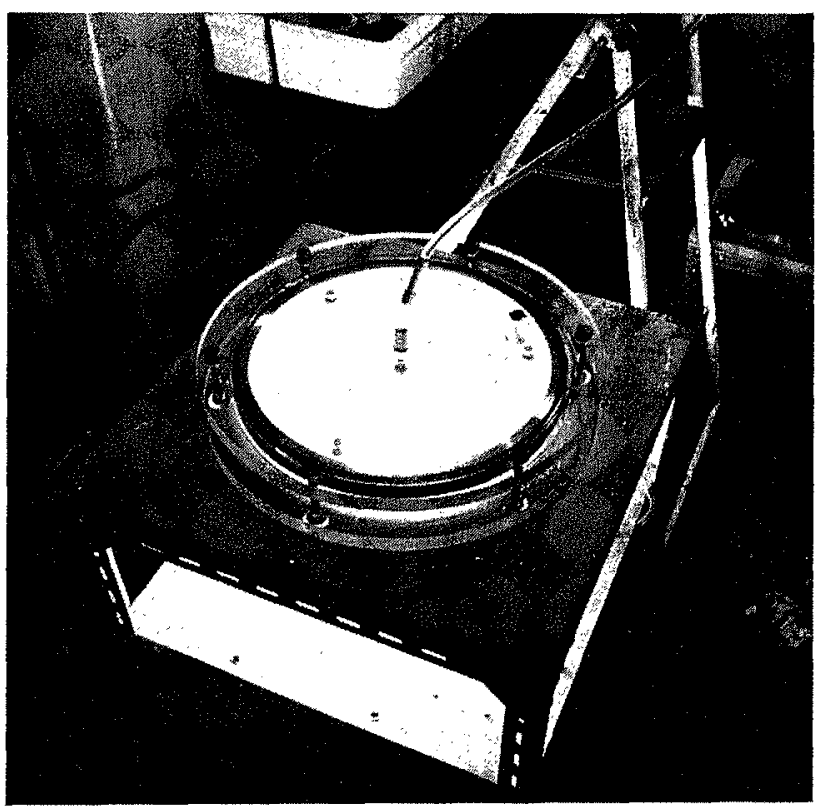

Pното 1

Modèle de Hele-Shaw. 


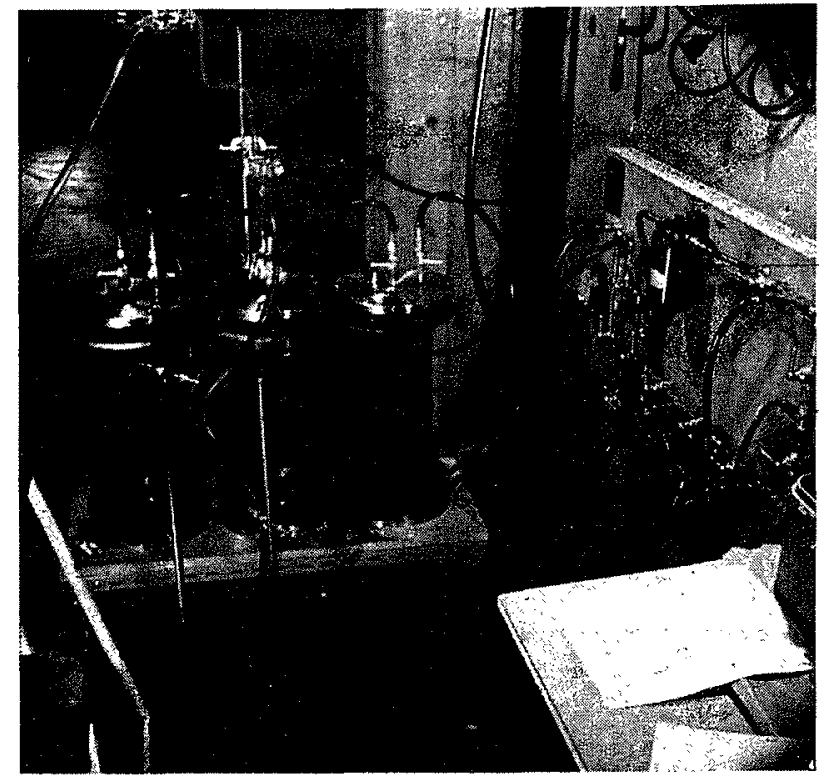

PHOTO 2

Installation des réservoirs contenant les liquides.

cylindres en laiton sur lesquels appuie le disque supérieur en écrasant des rondelles de caoutchouc. Ces cylindres permettent d'éviter que le disque intermédiaire soit soulevé à cause des différences de pression produites au cours de l'écoulement.

On alimente l'appareil à partir d'un tube en laiton de diamètre intérieur égal à $1 \mathrm{~mm}$ et situé au centre du disque inférieur. Pour assurer l'alimentation, nous avons utilisé l'appareillage suivant :

$1^{\circ}$ Deux pompes à engrenages Zénith qui sont entraînées par un moteur électrique couplé à un variateur de vitesse. On peut n'utiliser qu'une pompe ou les deux pompes montées en parallèle afin d'explorer une grande gamme de débits.

$2^{\circ}$ Un ensemble de trois réservoirs en Pyrex que l'on peut voir sur la photo $\mathrm{n}^{\circ} 2$. Le réservoir de droite contient, en haut, l'huile SAE 50 qui circule dans les pompes à engrenages et, en bas, de l'eau qui sert de liquide-tampon entre cette huile et l'huile contenue dans le réservoir du centre et destinée au modèle, car les réservoirs de droite et du centre sont réunis, en bas, par une canalisation.

Le réservoir de gauche contient, en haut, de l'huile qui vient des pompes à engrenages, et, en bas, de l'eau teintée de fluorescéine qui doit être injectée dans le modèle.

Nous allons, à présent examiner de quelle façon cet ensemble est connecté au modèle de Hele-Shaw.

\section{V. - DESCRIPTION DES EXPERIENCES}

Chaque expérience comporte deux phases:

$1^{\circ}$ Après avoir démonté et nettoyé le modèle, on remet en place les différentes pièces qui le composent. On procède au remplissage en huile en envoyant l'huile des pompes dans le réservoir de droite.

Le remplissage est effectué avec précaution afin d'éviter d'emprisonner des bulles d'air, jusqu'à ce que le niveau d'huile dans le modèle dépasse le dessus du disque intermédiaire.

$2^{\circ}$ La deuxième phase consiste à injecter, à un débit connu, l'eau teintée de fluorescéine dans le modèle.

\section{VI. - PRESENTATION DES PHOTOGRAPHIES}

Les photographies qui sont présentées ici correspondent à deux des expériences préliminaires qui ont servi à mettre au point le système de visualisation.

Les photographies ont été prises à l'aide d'un appareil Robot $(24 \times 36 \mathrm{~mm})$ placé verticalement au-dessus du modèle horizontal qui ètait éclairé par en dessous à l'aide d'une lampe fluorescente circulaire. L'obturateur était déclenché à intervalles réguliers par une minuterie, dont on faisait varier la période, en cours d'expérience, car la vitesse moyenne de déplacement du front diminue au cours du temps.

Comme l'huile employée est parfaitement transparente, l'eau apparaît en gris sur les photos.

Les photos 3 à 7 correspondent à un déplacement d'huile par de l'eau. La photo $\mathrm{n}^{\circ} 3$ a été prise au début de l'injection d'eau. Comme on le voit, le front a encore une forme presque circulaire. Mais les photos $n^{\circ} 4$ et $n^{\circ} 5$ montrent que ce front se déforme et présente un renflement qui s'accentue.

Sur la photo $n^{\circ} 6$, apparait nettement une digitation qui pousse sur le premier renflement. Elle est suivie d'une deuxième que l'on aperçoit sur la photo $\mathrm{n}^{\circ} 7$.

Pour nous rendre compte si la formation des digitations dépend bien du rapport des viscosités, nous avons également procédé à un déplacement d'eau par de d'huile, avec le même débit que celui du déplacement des photos 3 à 7 .

Nous avons vérifié que le phénomène présen- 
Pното 4

$7 \mathrm{mn} 9 \mathrm{~s}$ à partir du début de l'injection.

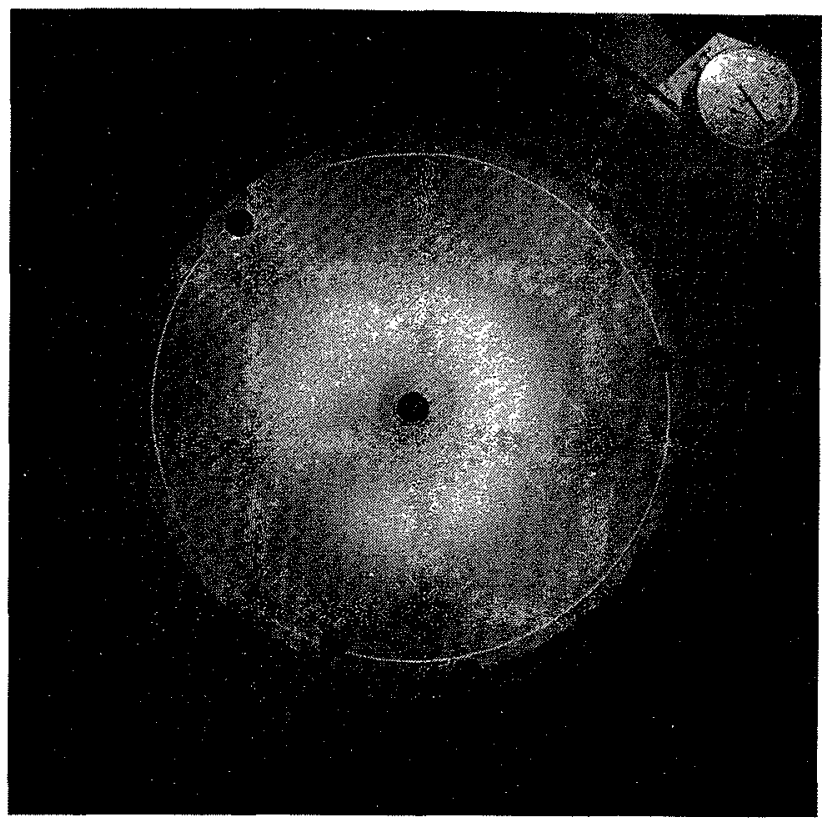

Photo 3

$2 \mathrm{mn} 29 \mathrm{~s}$ à partir du début de l'injection.

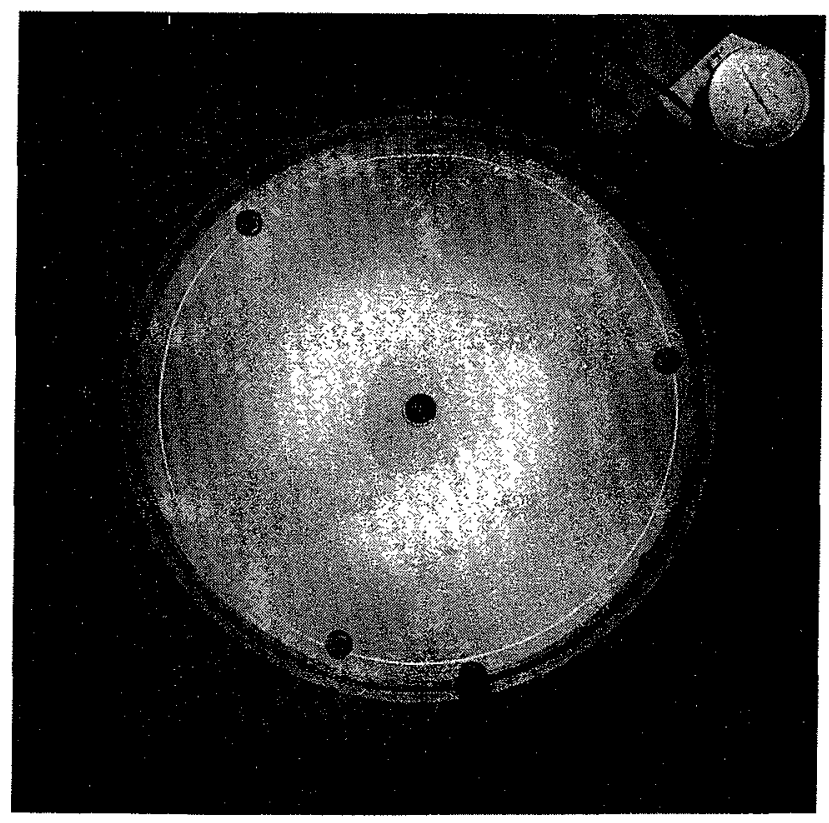

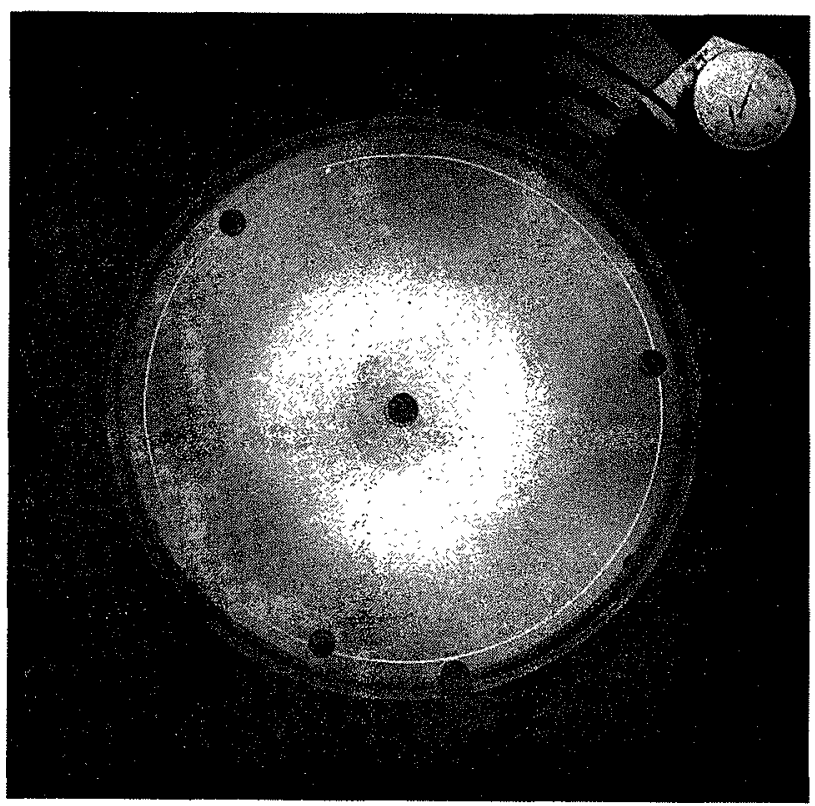

Pното 6

$13 \mathrm{mn} 22 \mathrm{~s}$ à partir du début de l'injection.

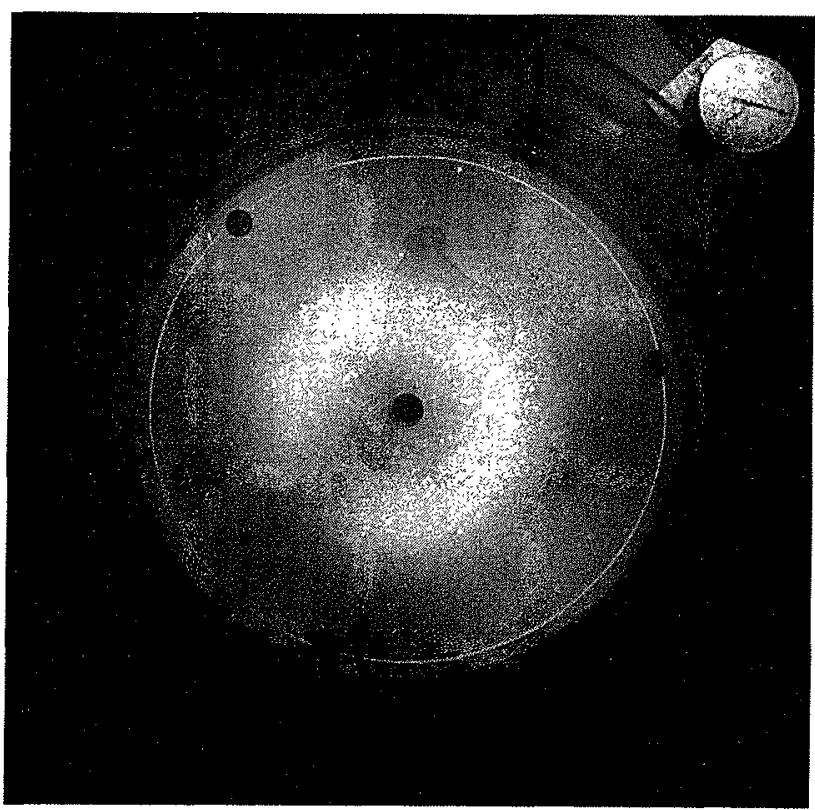

Рното 5

$10 \mathrm{mn} 0 \mathrm{~s}$ à partir du début de I'injection. 


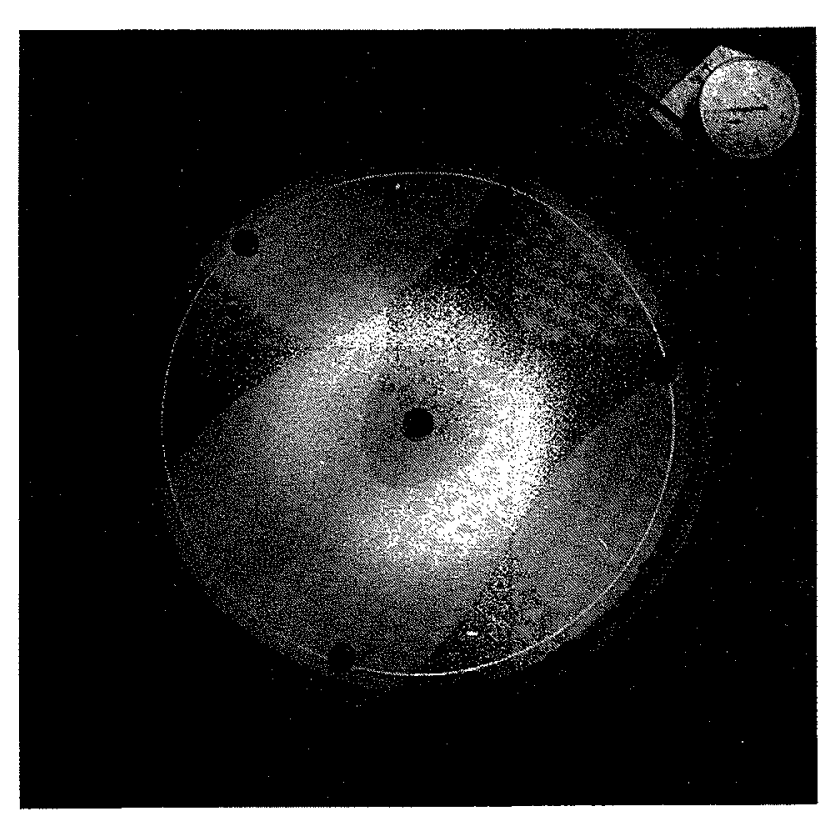

Рното $\bar{i}$

$15 \mathrm{mn} 50 \mathrm{~s}$ à partir du début de l'injection.

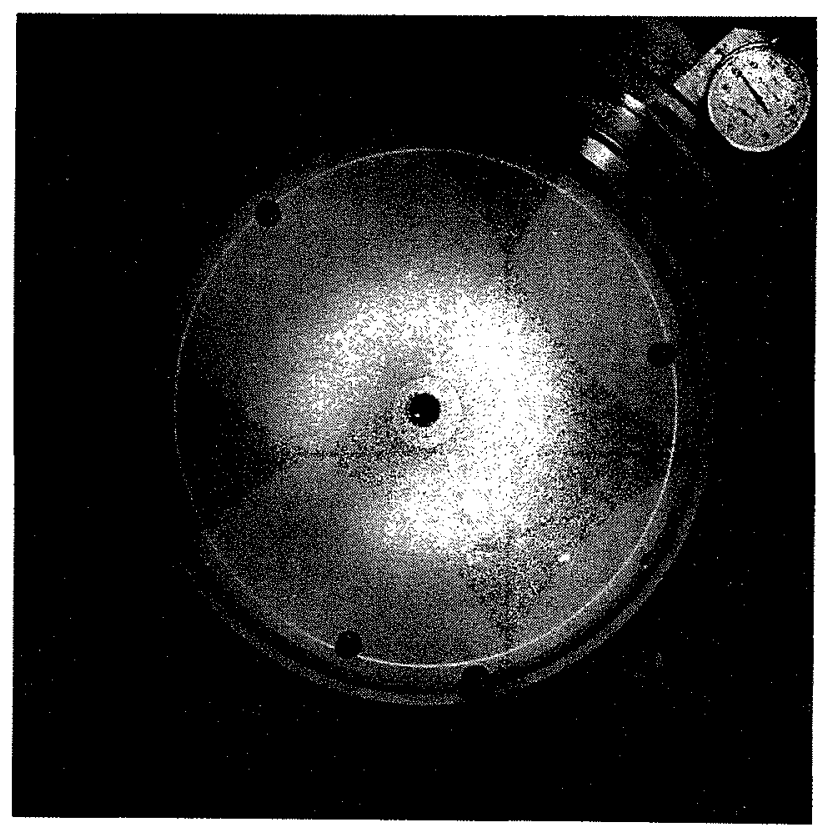

Photo 8

$2 \mathrm{mn}+5 \mathrm{~s}$ à partir du début de l'injection.

tait un caractère différent (photos 8 à 12). Le front n'a pas la forme circulaire de la théorie, car les plaques de verre sont des glaces du com- merce non retouchées et leur écartement n'est pas tout à fait uniforme, mais il se déplace plus régulièrement et ne présente pas de renflements.

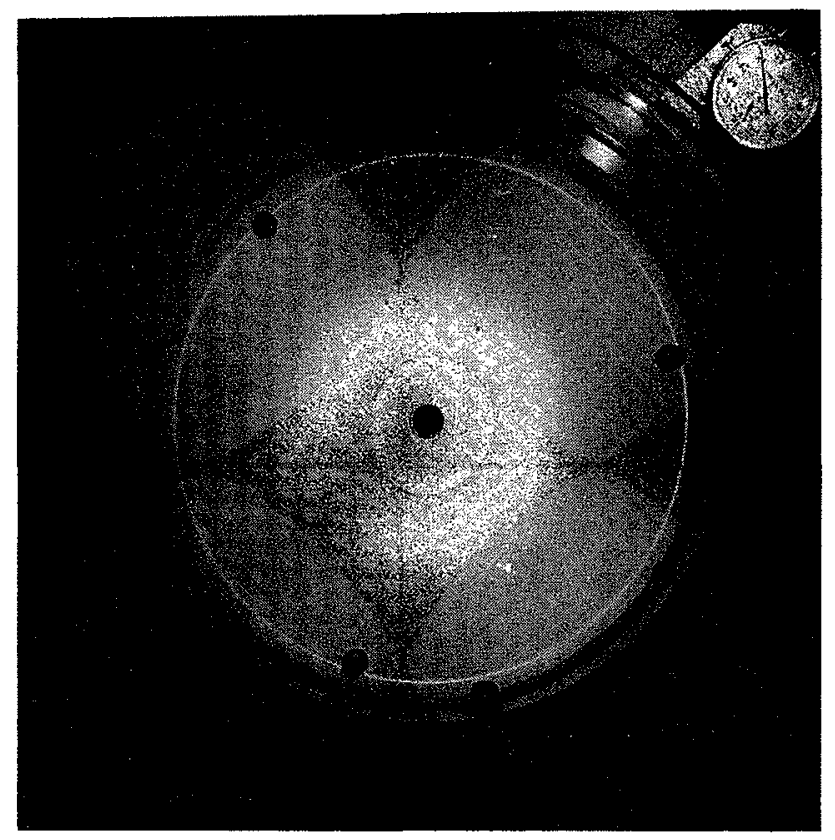

Photo 9

$8 \mathrm{mn} 49 \mathrm{~s}$ à partir du début de l'injection.

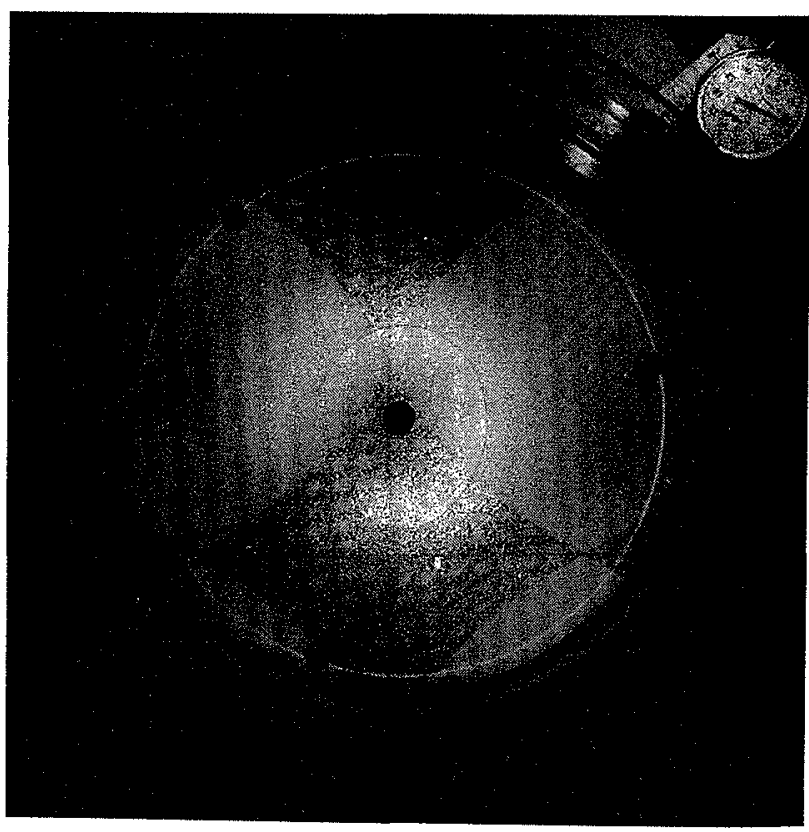

Pното 10

$17 \mathrm{mn} 10 \mathrm{~s}$ à partir du début de l'injection. 


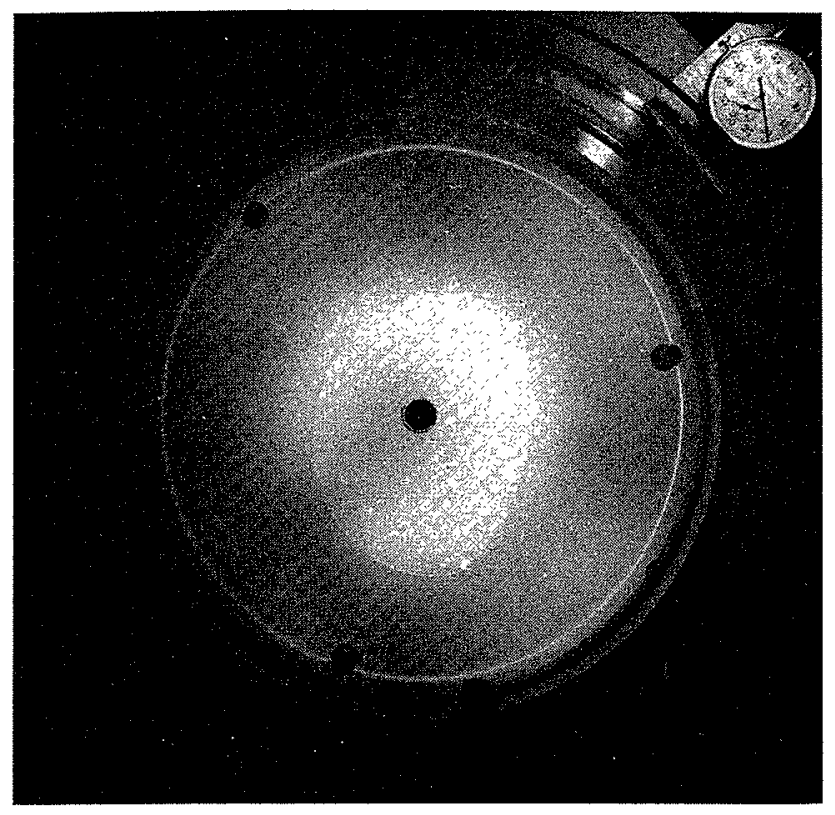

Photo 11

$30 \mathrm{mn} 19 \mathrm{~s}$ à partir du début de l'injection.

\section{VII. - CONCLUSION}

La visualisation des écoulements diphasiques entre deux plaques parallèles est plus facile que dans un milieu poreux où la matrice poreuse empêche, même si elle est transparente (billes de verre), de distinguer convenablement la forme du front.

On peut ainsi aborder l'étude qualitative des influences des divers paramètres : débits du puits, perméabilité (écartement des plaques), rapport des viscosités et tension interfaciale des

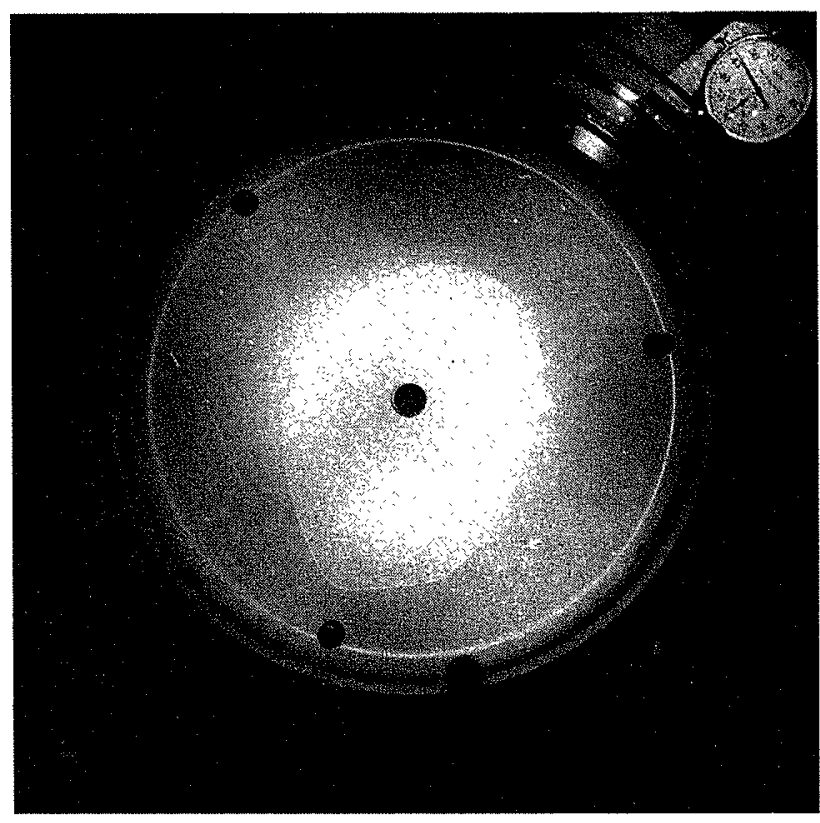

Pното 12

$40 \mathrm{mn} 46 \mathrm{~s}$ à partir du début de l'injection.

fluides, sur la forme et le nombre des digitations.

\section{BIBLIOGRAPHIE}

[1] Saffman (P.C.) et Taylon (G. L.). - The penetration of a fluid into a porous medium on Hele-Shaw cell containing a more viscous liquid, proceedings of the Royal Society, Series A, vol. 245, p. 312, 1958.

[2] Chuoke (R. L.), Van Meurs (P.) et Van der Poel. (C.). - The instability of slow, immiscible viscous liquidliquid displacement in permeable media, Transactions of the American Institute of Mining, Metallurgical and Petroleum Engineers, vol. 216, p. 188, 1959 .

\section{I S C U S S I O N}

Président : M. Chapouthier

M. le Président remercie M. LE Fur de son exposé et remarque la différence de comportement entre les deux injections représentées sur les clichés projetés : il y a une instabilité très marquée dans la première et une très légère dans la seconde, du côté opposé à la première.

M. LE FUR explique que la déformation du front elliptique correspondant aux dernières photographies projetées est due à la modification de l'écoulement à cause de la présence des cales.

M. le Président pense qu'on pouvait s'attendre à ce que la déformation de l'ellipse soit toujours dans le même sens.
M. Sackmann présente les deux remarques ci-après, concernant les instabilités ou irrégularités constatées par M. LE Fur, dans les expérierces qu'il vient de décrire.

" $1^{\circ}$ Formation et ébolution d'une source bidimensionnelle.

\& M. LE Fun attribue l'irrégularité croissante de la frontière de la source au défaut de planéité des glaces. Nous pensons, bien au contraire, que ces irrégularités sont dues à la nature et à la forme même de la source pseudo-ponctuelle. Au laboratoire de Mécanique des fluides de Strasbourg, nous avons non seulement réalisé des sources ponctuelles de symétrie de révolution parfaite. 
mais nous avons même produit des doublets (source + puits superposés) qui, dans le courant uniforme, donnent naissance à une ligne singulière circulaire quasi parfaite (image de l'écoulement plan autour du cylindre de révolution). $\gg$

* Toutes ces expériences (publiées plusieurs fois) ont été effectuées avec des glaces absolument ordinaires qui n'avaient aucune qualité optique spéciale.

* $2^{*}$ Instabilités se manifestant aux lignes de séparation.

« Nous pensons que ces instabilités sont dues à la nature intime et véritable de l'écoulement qui n'est plan et dérivant d'un potentiel de vitesses qu'en ce qui concerne la vitesse moyenne (débit), tandis qu'en réalité il existe entre les deux glaces un gradient de vitesse considérable (profil parabolique).

\& Nous pensons alors que, dans le mouvement relatif de deux liquides de viscosités différentes, le liquide de viscosité le plus élevée se comporte comme un solide par rapport au liquide de viscosité le plus faible. Là encore, nous avons montré à Strasbourg, bien avant 1939, qu'il se développe en amont d'un obstacle solide un système de tourbillons en fer à cheval responsable de toute une série d'effets dynamiques, dont notamment l'action d'affouillement. $\gg$

M. LE FUn répond a la première question de M. SACKMANN :

* La façon dont est constitué l'orifice d'alimentation peut, effectivement, influer sur le comportement au départ; et ceci peut être une des causes de la non-circularité du front, dans le cas dit favorable, c'est-à-dire le cas où l'on injecte de l'huile dans l'eau.

« Mais la première instabilité, dans le cas défavorable où l'eau est injectée dans l'huile, se produit lorsque le front est assez loin de l'orifice d'alimentation. 》

M. le Président précise que, dans l'esprit de M. LE FuR, l'instabilité de la frontière devrait s'atténuer au lieu de s'amplifier si elle était due à l'instabilité de la source d'alimentation.

M. Sackmann observe que, dans les expériences dont il a parlé, l'instabilité est proportionnelle à l'alimentation de la source et qu'elle augmente avec le diamètre de l'orifice.

M. le Président conclut, pour cette première question de M. Sackmañ, qu'il y aurait intérêt à confronter les expériences de M. LE Fur avec celles du Laboratoire de Strasbourg.

M. LE Fur répond à la deuxième question de M. SACKMANN :
« Je n'ai pas eu le temps de montrer comment on peut passer des équations de Stokes, auxquelles obéit la vitesse microscopique entre les plaques qui ne dépend pas d'un polentiel, anx équations de la vitesse moyenne qui, elles, en dépendent.

«Or, dans l'écoulement entre deux plaques parallèles, la vitesse moyenne loin d'un obstacle dépend d'un potentiel et devrait avoir une valeur non nulle à la paroi, alors que l'on a une condition d'annulation de la vitesse à la paroi de l'obstacle.

\& Dans ces conditions, il semble que les écoulements ne soient plus bi-dimensionnels, mais tri-dimensionnels à la paroi de l'obstacle. Si le front se déplace à une vitesse uniforme, on a, au voisinage du front, des profils de vitesse différents du profil parabolique dans les liquides 1 et 2 , et il se produit un phénomène analogue à celui que l'on observe à l'entrée d'un tube ou à l'entrée d'un écoulement entre deux plaques parallèles.

« Mais ces phénomènes sont d'autant plus faibles que le nombre de Reynolds est plus faible : dans les expériences exposées, ce nombre est égal à 1 pour l'eau, à $3 \mathrm{~mm}$ de l'orifice d'alimentation et l'effet de tri-dimensionnalité est probablement éliminé. 》

M. Legendre confirme l'importance des phénomènes tridimensionnels commentés par le Professeur Sackmann et qui seront mis en évidence par quelques séquences de l'un des films de M. WerLé.

L'O.N.E.R.A. a exploité l'écoulement en lame mince pour des recherches fondamentales sur la séparation en régime laminaire en s'attachant à définir le comportement de l'écoulement dans le voisinage des points de décollement et de recollement, où les forces d'inertie sont faibles et d'ailleurs négligées dans les théories qu'il s'agissait de confirmer.

Pour interpréter de tels écoulements et discuter les conditions de similitude, il faut non seulement vérifier que les phénomènes à rapprocher obéissent aux mêmes lois, mais s'assurer de l'identité des conditions aux limites et de l'unicité des solutions. Par exemple, bien qu'aucune loi connue ne fixe l'angle d'un décollement laminaire, l'expérience en lame mince n'a jamais révélé que des décolllements tangentiels ou normaux; mais il serait aventuré de prendre pour base cette constatation pour lever l'indétermination des problèmes de séparation. Les instabilités observées par les auteurs sont également des indications de défauts d'unicité des solutions ou d'extrême sensibilité aux conditions aux limites. 》

M. le Président remarque que l'intervention de M. L.EGENDRE fait la transition et i.l donne la parole à M. Werlé pour son exposé. 